\title{
Spatial frequency and the detection of temporal discontinuity in superimposed and adjacent gratings
}

\author{
GLEN SMITH \\ University of Melbourne, Parkville, Victoria 3052, Australia \\ EDWIN R. HOWELL \\ National Vision Research Institute of Australia, Carlton, Victoria 3053, Australia \\ and \\ GORDON STANLEY \\ University of Melbourne, Parkville, Victoria 3052, Australia
}

\begin{abstract}
The properties of the detecting mechanism involved in the resolution of temporal discontinuities of visually presented stimuli have been investigated in two experiments. In Experiment 1 , observers made judgments of discontinuity when superimposed presentations of sine-wave gratings of $1,3,6$, and 10 cycles/deg were presented for either a 20 - or a 200 -msec duration per presentation at a contrast of .1 and .4. Threshold separation for discontinuity detection indicated a linear increase as spatial frequency increased, with a lesser effect for longer duration exposure and higher contrast. Experiment 2 involved adjacent presentation of the second grating, and a small linear decrease in separation threshold as a function of increasing spatial frequency occurred. The effect of spatial frequency on temporal discontinuity detection is dependent on whether the second stimulus is superimposed or adjacent.
\end{abstract}

The ability of the visual system to resolve two presentations of a visual stimulus at brief interstimulus intervals (ISIs) is of considerable interest to visual scientists (Boynton, 1972). However, we believe that there is confusion in the literature, because what is essentially a detection process has been studied under different guises. For instance, this detection process has been studied variously as visual persistence in the context of visual information processing approaches to cognition (Turvey, 1978), as integration in relation to visual masking (Breitmeyer, 1980 ), and in relation to the suppression of gray-out during a saccadic eye movement (Corfield, Frosdick, \& Campbell, 1978).

As the majority of such studies conducted by psychologists have used tachistoscopes, there has been a need to develop a conservative threshold criterion for discontinuity detection. This has been necessitated by the presence of physical transient artifacts when there is switching from one channel to another. Typically, subjects have been instructed to ignore these transients and to detect gaps or blanks, and some

Requests for reprints should be directed to: Department of Psychology, University of Melbourne, Parkville, Victoria 3052, Australia. This project is funded by an ARGC grant to G. Stanley and G. Smith and by an NHMRC grant to E. R. Howell. The authors are grateful to Jacki Smith, Debbie Kiers, and Vicki Anderson for assistance in data collection. training is required to develop a consistent threshold criterion (Bowling, Lovegrove, \& Mapperson, 1979). We have developed video display equipment allowing much tighter threshold judgments for very brief temporal presentations.

The two experiments presented here were designed to investigate properties of the detecting mechanisms involved in the resolution of temporal discontinuity. Specifically, they are concerned with the effects of spatial frequency and spatial location on temporal discontinuity thresholds. They make use of customdesigned electronic circuitry and a cathode ray oscilloscope (CRO) with a low persistence phosphor (P15) effectively to eliminate any physical transient artifacts. Such apparatus enables the adoption of an artifact-free flicker criterion to detect discontinuity between two presentations of a visual stimulus.

Experiment 1 was concerned with the effect of spatial frequency on the judgment of discontinuity when two presentations of a sine-wave grating are superimposed in the same spatial location.

Previous studies by others using superimposed gratings have reported increases in threshold as a function of increasing spatial frequency. Meyer and Maguire (1977) used a gap criterion on repetitively recycled square-wave gratings, and Bowling et al. (1979) obtained a similar result with sine-wave gratings. Corfield et al. (1978) compared both sine- and square-wave gratings and found that there was a dif- 
ference between the two types of grating at lower spatial frequencies, with the square-wave gratings having lower critical blank durations than the sinewave gratings. Both grating types showed an increase in threshold as a function of increasing spatial frequency, the difference between them disappearing at the high spatial frequencies, at which the higher harmonics that enable differentiation of the two types of gratings were not visible. In their study, using gratings of .5 and 20 cycles/deg (cpd), they found no effect of contrast on critical blank duration. Bowling et al. (1979) reported that for gratings of 1 and $4 \mathrm{cpd}$ there was no effect of grating contrast, but for gratings of $12 \mathrm{cpd}$ there was a significant effect, with reduction in critical duration as contrast increased.

The studies reviewed here have used the highthreshold gap-detection task, and Bowling et al. $(1979$, p. 537) have suggested that persistence at all spatial frequencies may be due "primarily to the prolonged responses of sustained channels" in the visual system. The present experiments were concerned with the effects of spatial frequency on the detection of discontinuity using a flicker criterion, rather than on the absence of any residual trace of the first stimulus as required by a gap criterion. It was considered that this psychophysical task might relate more to transient processes associated with stimulus offset, and that if such was the case, then the relationship between spatial frequency of stimulus and the response measure might be different from that obtained by previous workers.

Experiment 1 of the present study obtained discontinuity threshold ISIs for spatially superimposed presentations of gratings of $1,3,6$, and $10 \mathrm{cpd}$, durations of 20 and $200 \mathrm{msec}$, and contrasts of .1 and .4. Experiment 2 was designed to obtain similar thresholds for gratings presented spatially adjacent to one another.

\section{EXPERIMENT 1}

Recent workers concerned with the relationship between spatial frequency and persistence (Bowling et al., 1979; Meyer \& Maguire, 1977) have employed a recycling procedure whereby the grating and blank interval are alternated for a number of cycles. Such a procedure may produce synchronization of periodic elements in a cellular ensemble, as has been suggested occurs in CFF studies by Pieron (see Boynton, 1972 , p. 204). However, given that the Lovegrove group appear to obtain similar functions with both the recycling procedure (Bowling et al., 1979) and a single trial of two presentations of the gratings (Lovegrove, Heddle, \& Slaghuis, 1980), the experiments reported here used the latter, more simple presentation mode.

\section{Method}

Subjects. The observers comprised 20 paid volunteer students, aged between 18 and 30 years, who had normal or corrected-tonormal vision. They were assigned randomly to either short $(20 \mathrm{msec}$ ) or long $(200 \mathrm{msec})$ stimulus duration conditions.

Apparatus. Vertical gratings with a sinusoidal luminance profile and independently variable spatial frequency and contrast were generated electronically on the screen of a Hewlett-Packard 1310A CRO. These gratings were generated by a gated function generator (Tektronix FG501) that could be turned on and off by a sequence of control pulses. When the grating was turned off, the display screen formed a uniform blank field having the same space-averaged luminance as the grating. The mean luminance of the display was $3.2 \mathrm{~cd} / \mathrm{m}^{2}$. Gratings were presented in a central rectangle of $10 \times 15 \mathrm{~cm}$ on the CRO screen at a distance of $114 \mathrm{~cm}$ from the subject. A 1-mm-thick horizontal black line bisected the screen as an aid to accommodation while minimizing fixationinduced afterimages from repetitive stimuli. In the first experiment, the gratings were presented on the complete screen. For Experiment 2, the control pulses to the function generator were adjustable to allow the presentation on either the left or right half of the screen at different times, as needed.

As the experiments required accurate and very brief stimulus durations, it was necessary to run the raster system generating the display much faster than is normally the case with this type of display. The line oscillator frequency was $600 \mathrm{kHz}$, and the frame refresh rate was $1 \mathrm{kHz}$. This enabled the generation of a complete new display field in $1 \mathrm{msec}$, so that all time intervals could be specified to an accuracy of $1 \mathrm{msec}$. The CRO had a P15 fast decay phosphor.

Procedure. The task was to indicate on each trial whether an uninterrupted presentation of a grating or some interruption during the presentation was perceived. The subject was seated in front of the screen and was asked to fixate the horizontal line with both eyes open. On most trials, a sinusoidal grating was presented twice: the first presentation was replaced by the blank screen for a variable interstimulus interval (ISI), and then the second presentation was superimposed on the same spatial location. The space-averaged luminance was constant at $3.2 \mathrm{~cd} / \mathrm{m}^{2}$ throughout these three conditions. On approximately $12 \%$ of the trials, the grating continued without interruption through the interstimulus interval. No transient flicker was associated with these catch trials as the stimulus was not physically altered. The subjects were instructed to say "yes" if they perceived an interrupted grating and "no" otherwise. Most subjects reported basing their judgments on the presence or absence of a "flicker" in the display. As the subjects said "yes" to less than $5 \%$ of the catch trials, no further analysis of false positives was carried out.

The ISI was adjusted by a staircase method, detailed below, to establish the threshold for discontinuity or "flicker" detection. Eight threshold values were found for each subject, at contrasts of .1 and .4 for spatial frequencies of $1,3,6$, and $10 \mathrm{cpd}$. For half the subjects, the duration of the pre- and postblank grating was $20 \mathrm{msec}$, and for the other 10 it was $200 \mathrm{msec}$. The order of the eight conditions was randomized among subjects. Each threshold was the mean of four values. Starting with a long ISI, at which the stimulus was clearly seen as two distinct presentations (150 msec for the short stimulus duration and $80 \mathrm{msec}$ for the long stimulus duration), the ISI was reduced on successive trials by $10 \mathrm{msec}$ until the subject first reported no flicker on two successive trials. A slightly shorter ISI (by about $20 \mathrm{msec}$, if possible) was taken as the starting value for an ascending staircase in 2 -msec steps, until the first occurrence of two successive "flicker" reports. A third and fourth value were found in a like manner, starting with a value well below the threshold $(10 \mathrm{msec}$ for the short stimulus duration and $1 \mathrm{msec}$ for the long stimulus duration) and stepping up and then down. The descending staircase was used first for half the conditions (randomized between subjects), and the ascending one was used first for the rest. 


\section{Results}

The mean thresholds, expressed as ISIs, for detection of grating discontinuity as a function of spatialfrequency, contrast, and stimulus duration are shown in Figure 1.

An analysis of variance showed all effects to be significant. The main effects and their associated $F$ ratios were: stimulus duration ( 20 vs. $200 \mathrm{msec}$ ), $\mathrm{F}(1,18)=23.38, \mathrm{p}=.0001$; contrast $(.1$ vs. .4$)$, $\mathrm{F}(1,18)=14.12, \mathrm{p}=.001$; spatial frequency $(1,3,6$, and $10 \mathrm{cpd}), F(3,54)=23.13, p=.0001$. The twoway interactions were: stimulus duration $\times$ contrast $[\mathrm{F}(1,18)=7.12, \mathrm{p}=.16]$, stimulus duration $\times$ spatial frequency $[F(3,54)=10.18, p=.0002]$, and contrast $\times$ spatial frequency $[\mathrm{F}(3,54)=7.89, \mathrm{p}=.0002]$. The threeway interaction, stimulus duration $x$ contrast $x$ spatial frequency, was also significant $[F(3,54)=4.08$, $\mathrm{p}=.011 \mathrm{]}$. Increasing contrast decreases the detection threshold for flicker, as does increasing stimulus duration or decreasing spatial frequency.

Using polynomial contrast on spatial frequency revealed that the linear component was significant for the main effect of frequency and its interactions

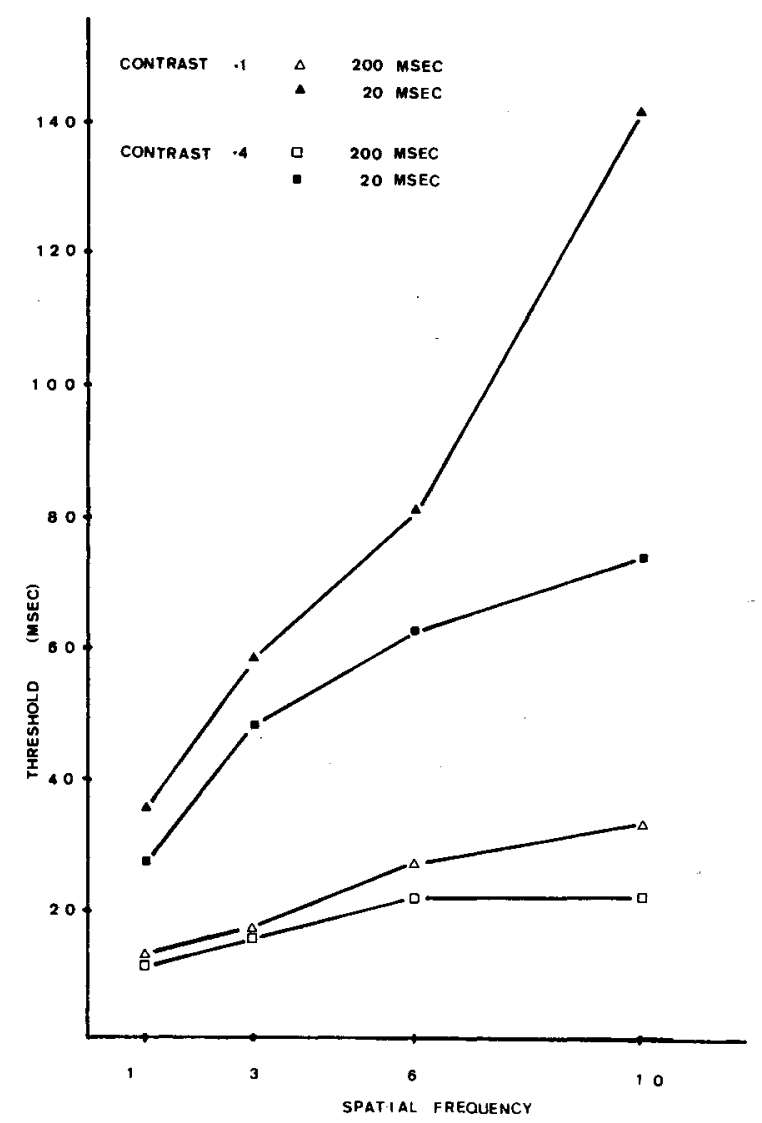

Figure 1. Mean ISI threshold (msec) for detection of an interrupted stimulus in two successive superimposed full-screen grating presentations as a function of spatial frequency for two contrasts and two stimulus durations. $(\mathrm{p}<.002)$, but neither quadratic nor cubic components approached significance $(p>.15)$. That is, the data are well described by straight lines, relating threshold to spatial frequency at both contrasts, both durations, and all four contrast $x$ duration levels used. These lines have different slopes for different contrasts and durations, and these two effects interact. Over the $1 \mathrm{log}$ unit range from 1 to $10 \mathrm{cpd}$, threshold was a linear rather than logarithmic function of frequency, since, for this data, a logarithmic transform of the spatial frequency effect introduced a significant quadratic component in the main effect and interactions. ${ }^{1}$

In showing that the threshold ISI for judgment of temporal discontinuity increases linearly with spatial frequency, the present results are in line with those obtained by Meyer and Maguire (1977) for squarewave gratings and by Bowling et al. (1979) for sinewave gratings. Two points are noteworthy: first, in the present results the thresholds are much lower than those obtained by the other workers with their "gap" detection criterion, and second, there is an interaction between spatial frequency and contrast which Bowling et al. (1979) did not find.

The finding that the effects of spatial frequency on the flicker discontinuity threshold are equivalent in magnitude but lower in absolute value than those obtained using a gap judgment does not immediately suggest that a different process is being studied with the different response measures. Given that the values obtained with our low spatial frequency stimulus are as short as $10-40 \mathrm{msec}$, there is a reasonable basis for suggesting that the processes are more akin to a detection system than to a persistence/short-term memory system.

\section{EXPERIMENT 2}

Spatial superimposition of the two presentations of a stimulus as used in Experiment 1 means that essentially an offset-onset relationship is studied. Such an array sets intrinsic limits on the lower bound for temporal separation, as it is not possible to study time intervals shorter than the stimulus duration of the first presentation. A number of experiments concerned with visual temporal discrimination have employed arrays in which the elements are not spatially superimposed (Di Lollo, 1980; Eriksen \& Collins, 1967). Such experiments allow onset-onset relationships to be studied and mean that the duration of the first presentation does not set any limit on the detection of the second stimulus, for it is possible to present a second stimulus while the first is still physically present. Moreover, the separate effects of "on" and "off" processes can be studied because it is possible in such displays to make stimulus offsets temporally coincident. 
Experiment 2 examines the effects of spatial frequency on discontinuity thresholds for sine-wave gratings presented spatially adjacent to one another. As separation ISIs of the order of $20-40 \mathrm{msec}$ were obtained at low frequencies in Experiment 1, the dependent variable reported in Experiment 2 was stimulus onset asynchrony (SOA). To use ISI with a stimulus duration of $200 \mathrm{msec}$ would have resulted in instantaneous judgments of discontinuity. Moreover, Boynton (1972) and Stanley, Howell, and Smith (1981) have argued the case for using SOA rather than ISI when one is considering temporal resolution in the visual system. Given that SOA is equivalent to ISI plus stimulus duration, it can be seen that they are comparable measures of discontinuity detection.

To explore further parameters of spatial frequency effects, some use was made of the fact that the adjacent presentation mode enables examination of onset-onset relationships. A $20-\mathrm{msec}$ stimulus followed at varying SOAs by a 20 -msec stimulus did not allow simultaneous offsets. Synchronous offsets were provided by having a $200-\mathrm{msec}$ grating followed on the adjacent side by a grating whose stimulus duration was $200 \mathrm{msec}$ less the SOA for discontinuity detection. This latter condition allows for a better manifestation of onset-onset effects than the 20-msec condition, in which the onset-onset effects are in a more complex relationship to offset.

\section{Method}

Subjects. Five other students were recruited as paid subjects for Experiment 2. All reported either normal or corrected-to-normal vision. They were experienced at judging discontinuity in gratings, having been subjects for tasks similar to those reported here.

Procedure. Stimulus onset asynchrony (SOA) thresholds for the detection of a discontinuous grating were found, using the method used in Experiment 1 for determining ISI thresholds in Experiment 1. Two stimulus types were used: adjacent $20 \mathrm{msec}$ and adjacent $200 \mathrm{msec}$. In both, a vertical sinusoidal grating was presented on the left half of the screen, followed by the right-half screen after a variable SOA. The relationship between the two halves of the screen was kept in spatial phase even with delay of presentation of the right half. In the 20 -msec condition, the grating on each half of the screen stayed on for $20 \mathrm{msec}$, so the two half-screen stimuli had asynchronous onsets and offsets. In the 200-msec condition, the left-half screen was presented for $200 \mathrm{msec}$, and the right half for $200 \mathrm{msec}$ less the SOA, giving synchronous of fsets.

Each subject provided 16 thresholds, 8 for the 20 -msec condition and 8 for the 200-msec condition, involving contrasts of .2 and .4 , and spatial frequencies of $1,3,6$, and $10 \mathrm{cpd}$.

\section{Results}

Mean SOA thresholds for discontinuity detection for the two stimulus types and two contrasts are plotted as a function of spatial frequency in Figure 2. An analysis of variance revealed significant effects of stimulus duration $[\mathrm{F}(1,64)=6.6, \mathrm{p}=.035]$, contrast $[F(1,64)=7.9, p=.006]$, and spatial frequency $[\mathrm{F}(3,64)=3.5, \mathrm{p}=.02]$, but no significant interactions $(\mathrm{ps}>.30)$. Once again, a polynomial contrast

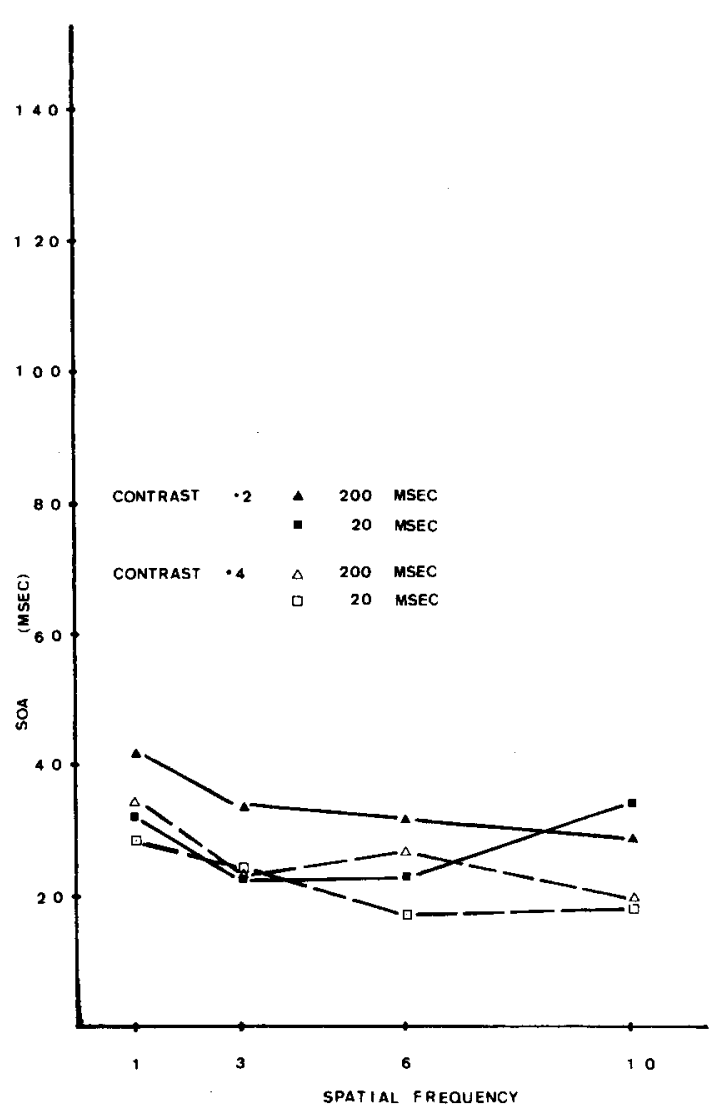

Figure 2. Mean SOA threshold (msec) for detection of an interrupted stimulus in left-half screen followed by adjacent righthalf screen grating presentations as a function of spatial frequency for two contrasts and two stimulus durations.

on spatial frequency revealed a significant linear component, but no quadratic or cubic component.

The most unexpected feature of these results is that there is a tendency for a decrease in discontinuity threshold as a function of increasing spatial frequency. Detection of temporal discontinuity for the adjacent presentations of sine-wave gratings clearly differs in this respect from superimposed presentation.

\section{DISCUSSION}

The experiments reported here have been concerned with discontinuity detection in successive presentations of sine-wave gratings. We believe that the data from these experiments represent the performance of temporal discontinuity detecting systems in the relatively early stages of the processing of visual information, rather than higher level shortterm memory processes of the type which are believed to form the basis of the commonly observed visual persistence phenomena (Turvey, 1978).

The main support for this interpretation comes from the very small absolute magnitude of the tem- 
poral intervals measured in our experiments. In both experiments, the minimum temporal intervals for the 1 -cpd grating were in the range of 10 to $40 \mathrm{msec}$, considerably less than the range of 100 to $250 \mathrm{msec}$ for persistence phenomena. The data for the effect of contrast in both experiments also support a concept of detector limitation. For all experimental conditions, an increase in contrast had the effect of decreasing the detectable interval. This is as expected for a simple detector model but has always been considered paradoxical for a simple sensory persistence model (Coltheart, 1980).

Considering the results of Experiment 1 in isolation, it could be argued that the low spatial frequencies reflect the operation of a transient detector mechanism, whereas the high-frequency results reflect the operation of a sustained persistence system. However, such an interpretation is not readily compatible with either the stimulus-duration effect or the contrast effect and is made less credible by the results of Experiment 2, which indicate that the order of magnitude of spatial frequency effects changes dramatically in the adjacent presentation mode. In the latter mode, high-spatial-frequency gratings have a marginally lower threshold than the low-spatialfrequency gratings. Taken together, these experiments imply that the detector mechanism(s) are operating in complex ways at this early stage of discontinuity detection. Indeed, it may be necessary to postulate at least two populations of temporal detectors to explain our results.

In Experiment 2, the basic 200-msec stimulus condition involved only asynchronous onsets, and the similarity of its curves to those of the 20 -msec condition (see Figure 2) shows that the presence of asynchronous offsets and decreased stimulus duration have little effect. This implies that the detector mechanism involved in Experiment 2 appears to be primarily concerned with onset rather than offset events.

The results obtained here indicate that the effect of spatial frequency on the resolution of two temporally separated presentations of a sine-wave grating critically depends on whether those two presentations are superimposed or spatially adjacent. This would suggest that caution is required in interpreting spatial-frequency effects in terms of transient and sustained mechanisms in the visual system as has been proposed by Bowling et al. (1979) and Di Lollo and Woods (1980, 1981). Judgment of discontinuity per se has been avoided in previous research (Bowling et al., 1979) because of its artifactual presence in experimental displays. However, given that judgment of discontinuity represents a strict criterion for maximum fidelity in the persistence of a stimulus, the relationship between the present results and the higher threshold measures of persistence used by others needs to be explored further. The story involving spatial frequency effects on various components of visual information processing is just beginning and promises to be more complex than current accounts allow.

\section{REFERENCES}

Bowling, A., Lovegrove, W., \& Mapperson, B. The effect of spatial frequency and contrast on visual persistence. Perception, $1979,8,529-539$.

Boynton, R. M. Discrimination of homogeneous double pulses of light. In D. Jameson \& L. M. Hurvich (Eds.), Handbook of sensory physiology (V11/4): Visual psychophysics. Berlin: Springer-Verlag, 1972.

BRE ITMEYER, B. G. Unmasking visual masking: A look at the "why" behind the veil of the "how." Psychological Review, 1980, 87, 52-69.

Coltheart, M. Iconic memory and visible persistence. Perception \& Psychophysics, 1980, 27, 183-228.

Corfield, R., Frosdick, J. P., \& Campbell, F. W. Grey-out elimination: The roles of spatial waveform, frequency and phase. Vision Research, 1978, 18, 1305-1311.

Di Lollo, V. Temporal integration in visual memory. Journal of Experimental Psychology: General, 1980, 109, 75-97.

Di Lolto, V., \& Woons, E. Duration of visible persistence in relation to range of spatial frequencies. Journal of Experimental Psychology: Human Perception and Performance, 1981, 7, 754-769.

Eriksen, C. W., \& Collins, J. F. Some temporal characteristics of visual pattern perception. Journal of Experimental Psychology, 1967, 74, 476-484.

Lovegrove, W., Heddle, M., \& Slaghuis, W. Reading disability: Spatial frequency deficits in visual information store. Neuropsychologia, 1980, 18, 111-116.

MeYer, G. E., \& Maguire, W. M. Spatial frequency and the mediation of short term visual storage. Science, 1977, 198, 524-525.

Stanley, G., Howell, E., \& Smith, G. Stimulus duration and grating persistence: An instance of the 100-msec rule? Perception \& Psychophysics, 1980, 28, 597-598.

TURVEY, M. T. Visual processing and short-term memory. In W. K. Estes (Ed.), Handbook of learning and cognitive processes (Vol. 5). Hillsdale, N.J: Erlbaum, 1978.

\section{NOTE}

1. The present results apply to the case in which there are fixed contrast levels. A reviewer has pointed out that equation for visibility over spatial frequency may change the nature of the function.

(Manuscript received October 20, 1980; revision accepted for publication October 29, 1981.) 